\title{
Digital Market using Blockchain Technology in Cloud Environment
}

\author{
Beena G Pillai, Madhurya J A, Dayananda Lal N
}

\begin{abstract}
The majority organizations associate blockchain technology with the financial services manufacturing, it has concealed use within the manufacturing, government, healthcare, and education sectors as well. The Block chain is an unlock and dispersed ledger. The Blockchain contract between two or more parties is confirmable and everlasting documentation. The Proposed System is smart market system, in this system Producers can sell the product directly in the market under the municipal counsil supervision or through online using block chain technology in cloud environment. The planned work is to intend a decentralized cloud server. We are powerful authentication where the information regarding all the products is encrypted. Those who have registered in the system come to know the product details about selling and purchasing. A highly developed method in cryptography can be used to address the issue of confidentiality, and some system could even monitor how much contact persons have. The blockchain technology is united with cloud computing to offer the enlarged transparency and efficient market system. The proposed research work can apply merkle tree in the designed algorithm on smart contracts performance optimization and automatic repair. We acquire benefits of the scattered confirmation security of the Blockchain technology for financial transaction verification and influence smart contracts for protected service organization. In this system bitcoin, cryptocurrencies or any other money transfer mechanism is used for transaction. Without a third party dealings may get more profit for both sides. Different types of on-site circumstances are collected and checked by installing sensors that are IoT devices in different services, and on-site calculating devices are restricted slightly if required.
\end{abstract}

Keywords: Blockchain, cloud computing, elegant contracts, IoT, merkle tree,

About four key words or phrases in alphabetical order, separated by commas.

\section{INTRODUCTION}

Blockchain is one of the most popular topics for discussion now. On the other hand, the majority of specialist considered the knowledge barely the component of Bitcoin, additional crypto-currencies or currency transmitted method. Blockchain tools is worldwide and be able used in additional regions, for instance, in IoT, WSN and mobile devices.

Revised Manuscript Received on April 30, 2020.

* Correspondence Author

Beena G Pillai*, Dept. of CSE, Gitam University, Bangalore, India. Email: beenagpillai.pradeep@gmail.com

Madhurya J A, Dept. of CSE, Gitam University, Bangalore, India. Email: madhurya72@gmail.com

Dr. Dayananda Lal N, Dept. of CSE, Gitam University, Bangalore, India. Email: dayanandlal@gmail.com

(C) The Authors. Published by Blue Eyes Intelligence Engineering and Sciences Publication (BEIESP). This is an open access article under the CC-BY-NC-NDlicense

(http://creativecommons.org/licenses/by-nc-nd/4.0/)

Blockchain technology, are taking the world by tempest basically due to the success of Bitcoin, have utilized in different areas through hasty progress, consequential in the dusk of a latest financial system. Newly, a broad variety of blockchain supported functions and examination appeared. Conversely, main producers have suitable and efficient method to organize, preserve and supervising their appliances, and as a result they cannot guarantee the consistency and safety of the relevancies. Here lots of reasons designed for, however the main significant one is the convolution of the blockchain technology.

Previously producers are managing regulations; they are unacquainted the force from the intricate main structure, Therefore they cannot protected to agreement with the potential errors. Additionally, seeing that deficient in proficient fundamentals, usually developers or teams regularly be unsuccessful to deal with the current circumstances of their method and cannot consequently categorize and take proper procedures to secure the method fault point in time. To make easy resolve the predicament, producers frequently have to contribute the energy to find out the important apparatus of the blockchain, faster than focus on formulate of production system. In opposition, the effort concerning these critical tools is complicated for the majority of developers or squad.

The improvement of block chain technology, which consent to business decentralized representation, opens new possibility for businesses to accomplish communication and build contracts. One of the tools is intending an alternate towards the predictable representation be the elegant dealing. Since a smart contract take out trust on an intermediated while set up production dealings, the customers create a contract carry out straight among every one. A smart contract, instead, can execute the similar host endowment proceedings sharing items, setting objectives, and bring together contributions - but devoid of an intermediate. Basically series an elegant agreement to implement the entire proceedings.

The functioning of smart contract is all digitized and takes out the need of an agent or a notary as a middleman. The two agreement parties contest to the stipulations of the convention set. The agreement is scheduled in a database and all the authorized reimbursement is made preset. The benefit in the agreement is digitized and disbursement is done through the digital or cryptocurrencies. The smart contracts offer the parties of the contract self-determination of self-sufficiency, endorsement, security, rapidity, accuracy.

The marketplace for a smart contract is segmented on the foundation of Block chain proposal, tools, and customer by region. 


\section{Digital Market using Blockchain Technology in Cloud Environment}

On the source of block chain proposal, the fragmentations further divided in to Bitcoin, side chains, NXT and Enthereum. Enthereum is the majority of sophisticated coding and processing of the smart contract and description for the large share followed by bitcoin and NXT.

On the basis of technology the fragmentation is done on the basis of Enthereum, Root stock, Name coin, Ripple among others.

Elegant dealings are considered along with executed by blockchains, and consequently they succeed to various blockchain's functionalities: They are undeniable, with the intention of a smart contract can never be distorted and nobody interfere with or crack an agreement. They're spread, the product of the contract is authenticated by everybody in the system. Allotments formulate it unattainable on behalf of an aggressor in the direction of power manages to discharge resources, while the entire applicants distinguish an endeavor and spot it as unfounded. Smart contracts make sure coarse catalog footpath, benefitting contribute chain funding in addition to sinking the threat of stealing and deception. Smart contracts can efficiently exist in spite of bazaar style or commodities sold.

\section{CONTRIBUTIONS}

\section{A.Implementation of de-centralized server:}

Centralized server is always easy source for introduces to launch attack because data is centralized, de-centralized server overcomes this issue where data can be distributed to multiple servers.

\section{B. Implementation of block chain technology:}

Block chain technology plays major role to preserve data privacy at the time payment mode, where data is distributed over multiple nodes. Elegant dealings be able to afford concurrent visibility is provide chains.

\section{C.Implementation of Smart Contract in Market:}

The Proposed System is smart market system, in these system farmers/ producers can sell the product directly in the market under the municipal counsil supervision. The most important thing is to attract new merchants. Out of the profit $80 \%$ will go to farmers/ producers and $20 \%$ running the cooperative society. By the time is completed the people will earn twice what they used to.

\section{RELATED WORK}

The Bitcoin protocol as well as its key equipment feature offered demands and weakness of the Bitcoin system and its most important expertise consist of the blockchain procedure, safety learning and clarification and recapitulate unwrap investigation and development for future research in Bitcoin security.

These distinctive features to the blockchain, such as isolation, protection, obscurity, delegation, and immutability, afford precious reimbursement to a range of field and subjects. Its investigates the relevance of blockchain barely begin through various restricted learning in the region, seeing that IoT, power, investment, healthcare, and government, so as to assistance suspiciously as of its achievement.
The perception of"Systematic Blockchain" was projected, which can be familiar with put up WSN among the involvement of stylish Cars, as nodes of the system. The regulation of block configuration and construction in the chain is projected; an arithmetical representation is formed to it. Approximation in the most favorable quantity of WSN nodes, the amount of associations connecting nodes, used for particular consistency ethics, was executed.

The essential feature for the accomplishment of blockchain supported proposal dealings are (i) How SPs' involvement is replicated taking place its quality of service (QoS) and (ii) How to incentivize SPs to make a payment possessions for instance work out/storage communications. In the competition formulation, a non supportive two period energetic entertainment is organized, wherever the primary point representation is the succeeding points representation and the contest connecting platform to catch the attention of patrons.

As a result, we endow with a stability of investigation, that provide a functional approaching interested in how much the repair excellence of blockchain based proposal influences the struggle linking proposals with the symmetry inducement approach used for SPs. Furthermore, our arithmetical investigation demonstrates with the aim of the symmetric inducement enlarges through comparative to the QoS of a blockchain based proposal although the inducement be converted into unconstructive if it supply an ever-increasing QoS among the quantity of contributed SPs.

\section{PROPOSED SOLUTION}

The proposed system used as a uniqueness chain equipment and smart contracts susceptibility recognition. The major intention of the projected scheme is ambiguity of block exploitation and runtime. In proposed systems use merkle tree to find the smart contract performance optimization and automatic repair. The proposed research work is to design a decentralized cloud server using Baas platform. The major concern in this work is not only transaction but also to protect the data in a secured manner. Bazar is executing smart indenture technology surrounded by a peer to peer advertise wherever customers can swap over cryptographic possessions on the blockchain in the structure of field.

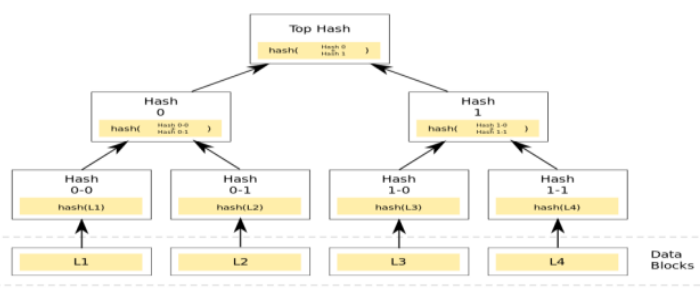

Fig1: Binary Hash Tree.

Merkle Tree can be used for escalating the strength and authority utilization of node is profitable for using extended blockchains. The existing systems are analyzed to find their concert metrics. In the proposed system a better performance will be tried to achieve so that it is practically deployable. 
In figure No : 01 the reliability of information block L2 can be demonstrated instantaneously if the tree previously include hash $0-0$ and hash 1 by hashing the data block and loops come together the end result through hash $0-0$ and then hash 1 and to end with measure capable of the result with the peak hash. Correspondingly, reliability of data blocks L3 able to demonstrate if the tree previously contain hash 1-1 and hash 0 . This preserve a benefit given that it is proficient to opening credentials positive in especially minute sequence blocks so that only undersized blocks have to be re-downloaded if they get injured. If the hashed document is very big, such a hash tree or hash list becomes comparatively big. But if it's a tree, single division preserve downloaded hastily, the reliability of the subdivision is able to checked, and then the downloading of records blocks can start.

To provide a flexible architecture for organizing cloud-blockchain technology and the transparency of blockchain deployment. To provide security for the data stored in the sub servers and an efficient way to retrieve data stored in the sub servers. To provide security of smart contracts. The most important thing is to attract new merchants. Out of the profit $80 \%$ will go to farmers/producers and $20 \%$ running the cooperative society. By the time is completed the people will earn twice what they used to.

Blockchain not only offers payback to companies but to their client base also. With more clear-cut approaches afford by elegant dealings, commerce be capable of maintain a realistic viewpoint to their clients while providing the security for the entire data carry out among the dealers. Since Blockchain provides clearness and simplicity to companies and to their whole supply chain. Proficient process of sharing related data like categorize and consignment statistics, transaction data and catalog data, companionship preserve to reduce all categories of reservations as well as improve their production. This direct to smaller quantity of occurrences deserves in allocation and enhance auction for the business.

Corporation preserves apply blockchain technology to improve their supply chain detectable. In this vision to facilitate frequently followed excluding incredibly complex to accomplish during several set of connections. By giving a proposal so as to set aside all associates to give information to a particular ledger, so business can increase various clearness in the direction of the entire category of services, crops and systems starting from provider in the direction of their clients. This assist a production headed extra precise representation of their position, assist them progress and increase on their interior commerce progression.

\section{HOW DOES BLOCKCHAIN WORK?}

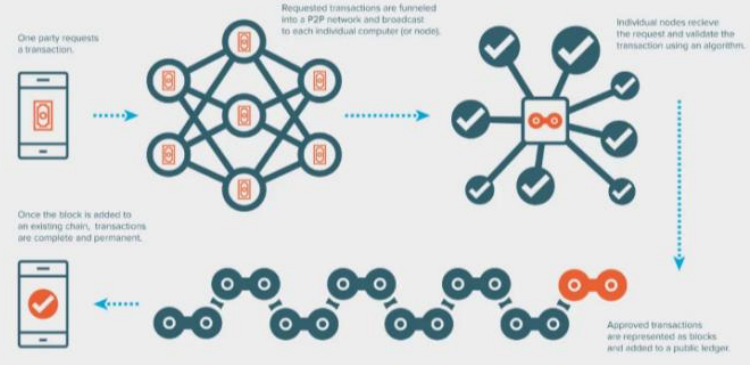

Fig2: Digital-marketing-industry.
Blockchain can also be used to continue pathway of data such as home records, voting records and medical in sequence. Each transaction is divided into blocks. These blocks will be confirmed by other users within the system. Blocks cannot be altered once they are confirmed. The recent confirmed blocks will be kept on adding to the already confirmed blocks.

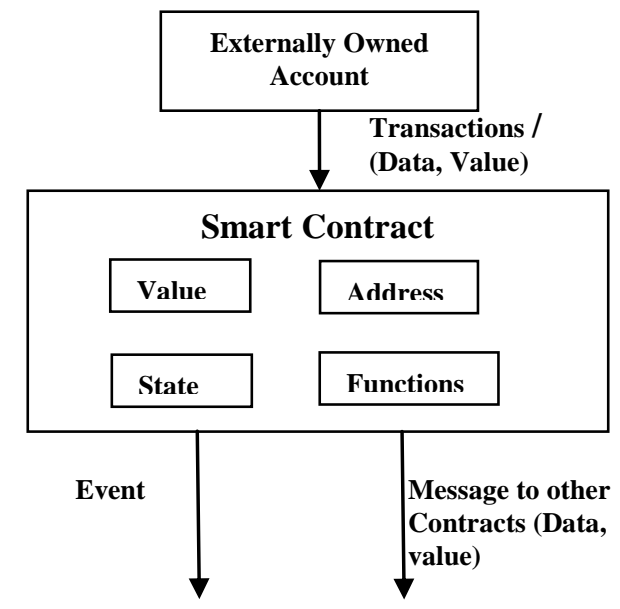

Fig3: System Architecture

Data and value transaction transferred through users owned account. All the transactions are in the form of encrypted format using private key and also it stored in cloud it seems. This encrypted data or message transferred to other contracts. Here the smart transaction is based on cryptocurrency by sing blockchain technology which will be benefit for the users and second party since the third party is not involved in the contract as well as all the dealings should be secured. New customers are also added in this contract.

\section{RESULT \& DISCUSSIONS}

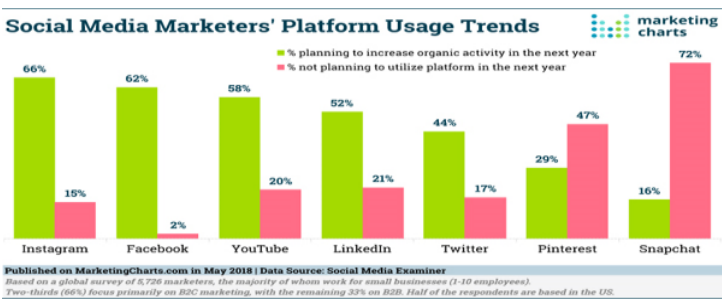

Fig4: MarketingCharts

Communal medium advertising information: Favored proposals and contented categories in 2018. The Social Media Examiner has given report which includes latest trends in social media, unveil with the aim of Instagram has outshine a dilapidated Twitter in general recognition, although B2B marketplace prefer to choose LinkedIn.

Facebook Released new report 'Theme to observe', enlisting the solution inclinations, U.S. distributors now have access to that latest report which was released by Facebook's called "Topics to Watch" on April, 2018, which includes longest rising discussion focus on the policy. A leakage appears at Face book's exploration used for pressure to promotion. 


\section{Digital Market using Blockchain Technology in Cloud Environment}

An indication within Facebook's achievable upcoming influencer advertising search engine tactics have be available, together with a recognized substance marketing tool. The most excellent existence and period to situation on Social Media:

The most victorious periods and existence for redeployment social media substances have been observed in innovative learning information starting Unmetric, presentation contradictory relocation engaging spots for Twitter, Facebook, and Instagram. Instagram formally Launched capability concerning consumer posts. All Instagram balance sheet now include this facility to re-share communal customer post to Instagram Stories, through a "Create a story with this post" characteristics.

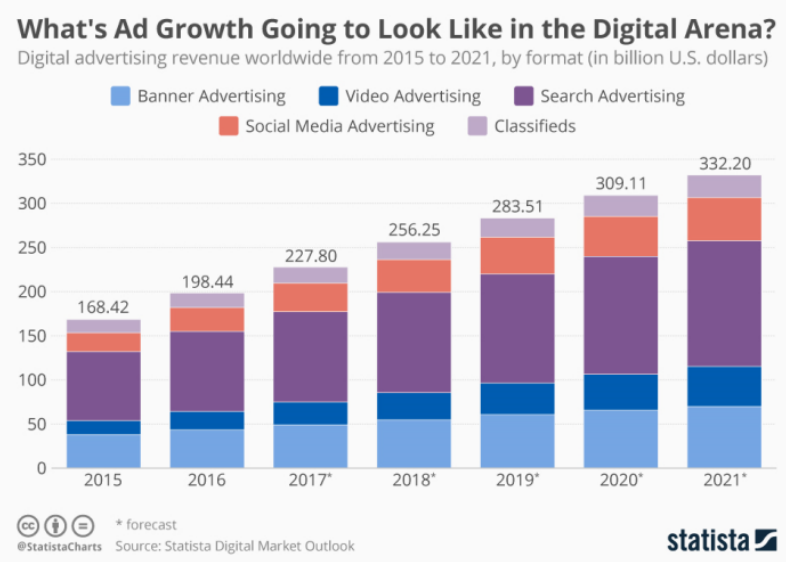

Fig5: Advantages of Digital-marketing

Digital marketing has the possibility to change the way to approach clients. At this time a number of actual patterns of how digital marketing strategies are serving business to accomplish customers and improve marketplace:

Ecommerce candle corporation, Nidhi improved sales by $714 \%$ more than a 3-month duration with social media. Everlastingly Diamonds, a Georgia ewelry company, be competent to approach its followers in support of less than \$1 per engagement over a period of year, which increased base transfer and important to genuine renovation. A snow motorcycle business advertising snow tools, construct \$96,664.98 worth of SEO results in just 5 months!

These situations are right evidence to the profit of digital advertising. By means of the correct approach and successful execution, little business preserves to enlarge their achievement and translations within a period of time.

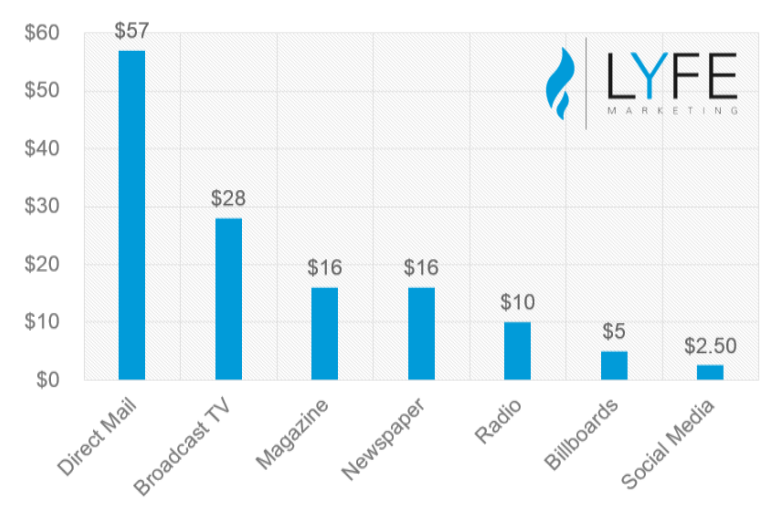

Fig6: digital-marketing
Consider this - a little production can interpretation more than 1,000 citizens to its goods and services for under \$3 with community medium. Whereas to identical revelation throughout straight packages regarding \$57 and throughout television advertisements expenses about \$28.

Whereas conventional promotion strategy also frequently approach with a variety of concealed expenditure, the simply cost to digital selling is point in time. Comfortable promotion and community media commitment acquire instance to effort their enchantment. Conversely, present digital marketing strategy such as compensate-for each-connect, demonstrate social media publicity that preserve construct more rapidly outcomes.

\section{CONCLUSION}

With the use of Smart contracts, worldwide deal resolves on a special viewpoint. Special characteristics of fabrication and allotment will take on a skilled outlook. Massive piles of formalities will be abolished while make sure that all varieties of deals are made in a protected, obvious and reliable system.

To make use of Smart contracts capacity comparatively new, but it have a lot of probable reimbursement container change the entire supply chains. Still confront by means of this organization and additional approach added in the future, it is extensively recognized with the purpose of all probable troubles determined hastily outstanding in the direction of the elasticity of the method.

\section{REFERENCES}

1. Mohamed Rahouti, Kaiqi Xiong, And Nasir Ghani "Bitcoin Concepts, Threats, and Machine-Learning Security Solutions", Digital Object Identifier 10.1109/ACCESS.2018.2874539 VOLUME 6, 2018

2. Joe Abou Jaoude And Raafat George Saade, "Blockchain Applications - Usage in Different Domains", Digital Object Identifier 10.1109/ACCESS.2019.2902501 VOLUME 7, 2019

3. Sergii Kushch, Francisco Prieto-Castrillo "Blockchain for Dynamic Nodes in a Smart City", 2019 IEEE 5th World Forum on Internet of Things (WF-IoT) 978-1-5386-4980-0/19/\$31.00 (2019 IEEE

4. Shancang Li , Senior Member, IEEE, Tao Qin, and Geyong Min "Blockchain-Based Digital Forensics Investigation Framework in the Internet of Things and Social Systems", IEEE TRANSACTIONS ON COMPUTATIONAL SOCIAL SYSTEMS, 2329-924X (C 2019 IEEE

5. Weilin Zheng, Zibin Zheng, Xiangping Chen, Kemian Dai, Peishan Li, And Renfei Chen "NutBaaS: A Blockchain-as-a-Service Platform" IEEE Access, Digital Object Identifier 10.1109/ACCESS.2019.2941905 VOLUME 7, 2019

6. Sergi Kushch, Francisco Prieto - Castrillo, "Block chain for Dynamic Nodes in Smart City" in 2019 IEEE 5th world Forum on Internet of things (WF-IoT)

7. Harry Halpin, Marta Piekarska "Introduction to Security and Privacy on the Blockchain”, 2017 IEEE European Symposium on Security and Privacy Workshops (EuroS\&PW)

8. S. Seebacher and R. Schritz, "Blockchain technology as an enabler of service systems: A structured literature review," Springer International Publishing, Cham, pp. 12-23, 2017.

9. R. C. Merkle, “'A digital signature based on a conventional encryption function," in Proc. Conf. Theory Appl. Cryptograph. Techn. Springer, 1987,pp. 369_378.

10. Merkle Patricia Tree. [Online]. Available: https://github.com/ethereum/ wiki/wiki/Patricia-Tree

11. P. McCorry, S. F. Shahandashti, and F. Hao, “'A smart contract for boardroom voting with maximum voter privacy," in Proc. Int. Conf. Financial Cryptogr. Data Secur. Springer, 2017, pp. 357_375.

12. A Reliable Bridge Between Smart Contracts and the Internet. [Online] Available: https://github.com/oraclize 
13. A. Chakravorty, T. Wlodarczyk, and C. Rong, "Privacy preserving data analytic for smart homes," 2013 IEEE Security and Privacy Workshop, pp. 23-27,, May 2013.

14. H. Menashri and G. Baram, "Critical infrastructures and their interdependence in a cyber attack the case of the u.s," Military and Strategic Affairs, vol. 7 no.1, pp. 99-122, 2015.

15. R. Roman, J. Zhou, and J. Lopez, "On the features and challenges of security and privacy in distributed internet of things," Computer Networks, vol. 57, no.10, pp. 2266-2279, 2013.

\section{AUTHORS PROFILE}

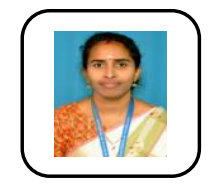

Beena G Pillai received the B.Tech degree in Computer Science \& Engineering from Acharya Nagrajuna University, Guntur, in 2012, the M.Tech degree in computer science and Engineering from Jawaharlal Nehru Technological University, Anantapur, in 2015. She is currently working as an Assistant Professor in the Department of Computer Science and Engineering, Gitam University, Bangalore. Her current research focuses on the security in Block chain technology, Cloud Computing and Cyber Security

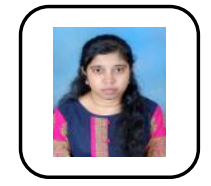

Madhurya J A received the B.E degree in Information Science \& Engineering from Visvesvaraya Technological University, Belgaum in 2011, the M.Tech degree in computer science and Engineering from Visvesvaraya Technological University, Belgaum, in 2017. She is currently working as an Assistant Professor in the Department of Computer Science and Engineering, Gitam University, Bangalore. Her current research focuses on the security in Cloud Computing, Cyber Security, IoT.

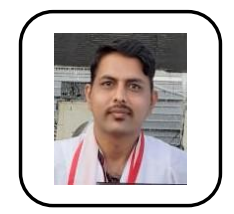

Dr. Dayananda Lal N Assistant Professor in CSE Department, GITAM School of Technology, Bengaluru campus. He has published three research papers "Porting presentation Layer to ensure network security in mobile devices", "configuring a secure wireless network using GNS3" and "Protective and Efficacious Research interest area is Cyber Security. 